\title{
Finiteness conditions in soluble groups and Lie algebras
}

\section{lan N. Stewart}

\begin{abstract}
We prove some new theorems and reprove some old ones about finitely generated soluble groups and Lie algebras by a uniform method. Among the applications are Gruenberg's Theorem on Engel groups, for which we obtain a very short proof; and the Milnor and Wolf polynomial growth theorem. It is shown that a finitely generated soluble group with all 2-generator subgroups polycyclic is itself polycyclic, and that a finitely generated soluble Lie algebra, all of whose inner derivations are algebraic, is finite-dimensional. This last result enables us to give a partial answer to a question of Jacobson.
\end{abstract}

The aim of this note is to illustrate a procedure for proving a number of theorems about finiteness conditions in soluble groups, based on quite simple considerations centred around the module-theoretic methods of Hall $[5,6,7]$. By virtue of the results of Amayo and Stewart [1] the procedure also applies to Lie algebras. Some of these theorems are well known, although others seem to be new. Among the former are:

(a) the theorem of Gruenberg [3] that finitely generated soluble Engel groups are nilpotent,

(b) the Lie algebra analogue of (a), also due to Gruenberg [3],

(c) the theorem of Milnor [9] and Wolf [10] that finitely generated soluble groups with polynomial growth are nilpotent-by-finite.

Received 20 February 1973. 
Our proofs of $(a)$ and (b) are very short, and use very little machinery: they are more conceptual than those of Gruenberg, which rely on properties of basic commutators. The proof of (c) is just a tactical variant of that given by Bass [2] and in consequence will be given in outline only. Our procedure is presumably 'folklore': in particular it seems that $\mathrm{Hall}$ has given in lectures a proof of (a) roughly along the same lines. However it is interesting that the same method proves such a variety of results.

\section{Notation}

We let $\underline{\underline{A}}, \underline{\underline{F}}, \underline{\underline{G}}, \underline{\underline{N}}, \underline{\underline{P}}$ denote the classes of abelian, finite, finitely generated, nilpotent, and polycyclic groups. We use Hall's closure operations $Q$ and $E$ : for any class $\underline{\underline{X}}$ of groups $Q \underline{\underline{X}}$ consists of $a l l$

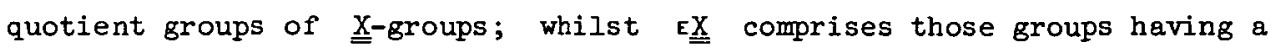
finite series

$$
1=G_{0} \triangleleft G_{1} \triangleleft \ldots \triangleleft G_{n}=G
$$

with each factor $G_{i+1} / G_{i} \in \underline{\underline{X}}$. Thus $E \underline{\underline{A}}$ is the class of soluble groups. If $\underline{\underline{X}}$ and $\underline{\underline{Y}}$ are group classes then $\underline{\underline{X Y}}$ denotes the class of

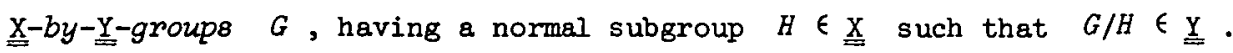

Let $\underline{X}$ and $\underline{\underline{Y}}$ be classes of groups satisfying

(i) all finitely generated $\underline{\underline{x}-g r o u p s ~ a r e ~ p o l y c y c l i c-b y-f i n i t e, ~}$

(ii) $\underline{\underline{Y}}$ is Q-closed.

Suppose we wish to prove that all finitely generated soluble $\underline{\underline{y}}$-groups are X-groups. Then we may try the following

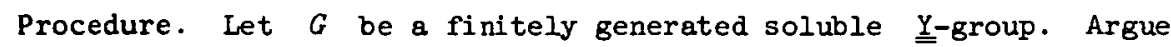
by induction on the derived length of $G$. If $A$ is the last nontrivial term of the derived series of $G$, then $A$ is abelian and normal in $G$, whilst $G / A$ is polycyclic-by-finite by induction. Therefore $G$ lies in the class $\underline{\underline{G}} \cap \underline{\underline{A P F}}$, which is studied by Hall $[5,6,7]$. In particular $A$ is a module for the integral group ring of the pF-group $G / A$, and $G$ satisfies the maximal condition for normal subgroups. This is a very strong condition, and often suffices to carry out the induction step. 
2. The theorems of Gruenberg, Milnor and Wolf

If $G$ is a group and $g, h \in G$ we write

$$
(g, h)=g^{-1} h^{-1} g h
$$

for the group commutator, and define recursively

$$
\left(g,{ }_{n+1} h\right)=\left(\left(g, n^{h}\right), h\right) \text {. }
$$

Then $G$ is an Engel group if for each $g, h \in G$ there exists $n=n(g, h)$ such that $\left(g, n^{h}\right)=1$.

To prove Gruenberg's Theorem we argue as above, with $\underline{\underline{X}}=\underline{\underline{\mathbb{N}}}$, and $\underline{\underline{Y}}=$ the class of Engel groups. By induction we may assume that our group $G$ lies in $\underline{\underline{G}} \cap \underline{\underline{A N}}$. To prove $G$ nilpotent it is sufficient to show that the abelian normal subgroup $A$ lies in the hypercentre of $G$, for then $G$ is hypercentral (since $G / A$ is nilpotent) and finitely generated, and hence $G$ is nilpotent. If this is not the case we may quotient out the intersection of the hypercentre with $A$. We may then assume, for a contradiction, that $A$ contains no nontrivial element centralised by $G / A$.

Let $N=G / A$ and argue by induction on the length (necessarily finite) of a cyclic series for $N$ that $A$ contains a nontrivial $N$-invariant element. This is clear if $N=1$. Otherwise we can find $K \triangleleft N$ such that $N / K$ is cyclic, $K$ has smaller cyclic length than $N$, and $N=\langle K, x\rangle$ for some $x \in N$. By induction there is an element $a$ of $A, a \neq 1$, which is invariant under $K$. Consider the subgroup $T$ of $A$ generated by all conjugates $a^{x^{i}}$ of $a$ by a power of $x$. This is clearly invariant under $x$. It is centralised by $K$, since if $k \in K$ then

$$
a^{x^{i} k}=a^{x^{i} k x^{-i} \cdot x^{i}}=a^{x^{i}} \text {. }
$$

By the Engel condition $\left(a, t^{x}\right)=1$. Let $t$ be smallest with this property. Then $\left(a, t_{t-1} x\right) \neq 1$, lies in $T$, and is centralised by $x$. Hence it is centralised by $N$. This is a contradiction, and Gruenberg's Theorem is proved. 
A very similar argument gives the Lie algebra version of the theorem.

The proof of the Milnor and Wolf Theorem follows the same lines, but is harder. We take $\underline{\underline{Y}}$ to be the class of groups with polynomial growth, $\underline{\underline{X}}=\underline{\underline{N F}}$. By Milnor [9], Lemma 1, we have $A$ finitely generated as an abelian group (which at once makes $G$ polycyclic). Since subgroups of finite index in finitely generated groups are also finitely generated we may assume $G / A$ nilpotent. Induction on the cyclic length of $G / A$, arguing as in Bass [2] p. 605 and using his Lemma 2 (which, as he remarks, is the essential point of the proof) completes the induction step.

\section{0 ther results}

The theorems of this section may all be proved by the same method.

THEOREM 1. Let $G$ be a finitely generated soluble group, alz of whose 2-generator subgroups are polgcyclic. Then $G$ is polycyclic.

Proof. We use the standard procedure, with $\underline{\underline{Y}}$ the class of groups all of whose 2-generator subgroups are polycyclic, and $\underline{\underline{x}}=\underline{\underline{P}}$. With the usual notation, we may assume that $P=G / A$ is polycyclic, and that $A$ is nontrivial, having no nontrivial $P$-invariant subgroup which is finitely generated as an abelian group.

We show by induction on a cyciic series for $P$ that on the contrary such a subgroup exists. Take $K \triangleleft P$ with $P / K$ cyclic, $P=(K, x)$. There is a nontrivial subgroup $B=\left\langle b_{1}, \ldots, b_{t}\right\rangle$ of $A$ which is $K$-invariant. Now each $\left\langle b_{i}, x\right\rangle$ is polycyclic, so that each $b_{i}$ lies inside an $x$-invariant subgroup $T_{i}$ of $A$ which is finitely generated as an abelian group. Thus $B$ is contained in $T_{1} \ldots T_{t}$, a finitely generated $x$-invariant group. Let $C$ be the product of the conjugates of $B$ under powers of $x$. Then $C$ is finitely generated and $x$-invariant. But if $y$ is any power of $x$ then

$$
B^{y K}=B^{K y}=B^{y}
$$

so that $C$ is also $K$-invariant, hence $P$-invariant. This completes the induction, and the resulting contradiction proves the theorem.

A similar argument yields: 
THEOREM 2. Let $L$ be a finitely generated soluble Lie algebra, all of whose 2-generator subalgebras are finite dimensional. Then $L$ is finite dimensional.

Examples mentioned by Golod [4] p. 103 (footnote) show that the hypothesis of solubility cannot be omitted from Theorems 1 and 2 .

Define the Lie algebra $L$ to be algebraic if every inner derivation satisfies some polynomial equation (which is allowed to vary from element to element). Then our procedure easily yields:

THEOREM 3. Every finitely generated soluble algebraic Lie algebra is finite dimensional.

Again an example of Golod [4] shows that we cannot omit the hypothesis of solubility.

COROLLARY 4. A Zocally soluble algebraic lie algebra is locally finite.

We can apply Theorem 3 to a question of Jacobson [8] p. 196. In Exercise 17 he states:

"Conjecture (probably false and probably true under additional hypotheses): If the restricted lie algebra $L$ of characteristic $p$ is finitely generated, and every element of $L$ is algebraic in the sense that there exists a non-zero p-polynomial $\mu_{a}(\lambda)$ such that $\mu_{a}(a)=0$, then $L$ is finite dimensional."

For the relevant definitions see Jacobson [8] pp. 185-194.

We show that the conjecture is true if, in addition, $L$ is required to be soluble. For in any restricted Lie algebra we have the equation (Jacobson [8] p. 188)

$$
\left[b, a^{p}\right]=[b, \underbrace{a, \ldots, a}_{p}]
$$

and in consequence the inner derivation induced by $a$ is algebraic (in our sense) if it is algebraic (in Jacobson's sense). Theorem 3 is now applicable, and for completeness we state:

THEOREM 5. If $L$ is a finitely generated soluble restricted lie algebra of. characteristic $p$, and if every element of $L$ is algebraic in 
the sense of Jacobson, then $L$ is finite-dimensional.

\section{References}

[1] Ralph K. Amayo and Ian Stewart, "Finitely generated Lie algebras", $J$. London Math. Soc. (2) 5 (1972), 697-703.

[2] H. Bass, "The degree of polynomial growth of finitely generated nilpotent groups", Proc. London Math. Soc. (3) 25 (1972), 603-614.

[3] K.W. Gruenberg, "Two theorems on Engel groups", Proc. Cambridge Philos. Soc. 49 (1953), 377-380.

[4] Е.С. Голод [E.S. Golod], "О ннль-алгебрах н финитно-аппронсимируемых p-группах" [On nil-algebras and finitely approximable p-groups], Izv. Akad. Nauk SSSR Ser. Mat. 28 (1964), 273-276; Amer. Math. Soc. Trans 2. 48 (1965), 103-106.

[5] P. Hall, "Finiteness conditions for soluble groups", Proc. London Math. Soc. (3) 4 (1954), 419-436.

[6] P. Hall, "On the finiteness of certain soluble groups", Proc. London Math. Soc. (3) 9 (1959), 595-622.

[7] P. Hall, "The Frattini subgroups of finitely generated groups", Proc. London Math. Soc. (3) 11 (1961), 327-352.

[8] Nathan Jacobson, Lie algebras (Interscience [John Wiley \& Sons], New York, London, 1962).

[9] John MIInor, "Growth of finitely generated solvable groups", $J$. Differential Geometry 2 (1968), 447-449.

[10] Joseph A. Wolf, "Growth of finitely generated solvable groups and curvature of Riemannian manifolds", $J$. Differential Geometry 2 (1968), 421-446.

Mathematics Institute,

University of Warwick,

Coventry, England. 